\title{
COMPOSICIÓN CORPORAL DE BAILARINES COSTARRICENSES DE DANZA CONTEMPORÁNEA
}

\author{
Yamileth Chacón Araya \\ María del Rocío Valverde Hidalgo
}

Recibido 16-VIII-2002

- Aceptado 12-XI-2002

\section{Introducción}

La danza desde tiempos remotos ha sido vivida y utilizada por el ser humano como un medio de comunicación, empleando sentimientos y elementos como el dolor o el amor como estímulos para la expresión interna-externa, verbal y no verbal, de los acontecimientos físico-mentales. A su vez, es un medio que se nutre de la constante capa-

Resumen: El propósito de esta investigación fue conocer las características antropométricas y de composición corporal de bailarines costarricenses. En Costa Rica no existen normas de composición corporal que permitan una selección objetiva de los bailarines que desean integrarse a las diferentes compañías de danza. En este estudio participaron voluntariamente 91 bailarines masculinos ( $\mathrm{n}=17)$ y femeninos $(\mathrm{n}=73)$, con edades entre los 17 y 35 años de edad, los cuales fueron agrupados en categorías profesional (P), semiprofesional (SP) y aficionado (A). Por medio de un análisis de varianza de una vía se encontró que las bailarinas $\mathrm{P}$ presentaban \% GC significativamente menores $(\mathrm{p}<0.05)$ que las bailarinas SP y A. También se encontró que las bailarinas de 17-21 años presentaban \% GC mayores que las bailarinas de 22-26, 27-31 y 32-35 años. Se presentan normas de composición corporal para bailarinas y bailarines costarricenses.

Palabras clave: Composición Corporal, Bailarines, Danza. cidad de creación de los seres vivientes y danzantes. La danza, a través de nuevas formas y lenguajes de expresión, ha ido aumentando la versatilidad y el vocabulario del movimiento humano tanto en su plasticidad como en su realismo mágico.

Por lo tanto, la danza ha permitido desarrollar y enriquecer las principales condiciones físico-mentales del danzante, tales como la estética corporal, la visión y la percepción justa de las formas, el sentido del ritmo, la coordinación de las fuerzas, la ligereza, la resistencia, y el equilibrio (Bougart, 1966). Al respecto, Minton y Beckwith (1986) mencionan que un bailarín se debe entrenar continuamente en el aspecto físico y técnico para mejorar la calidad del movimiento. Sin embargo, se ha señalado la necesidad de examinar detenidamente al bailarín y comprender todas sus posibilidades y limitaciones, debido entre otros aspectos, a la variabilidad de su constitución física (Dumond, 1954).

Uno de los factores que influyen en la estética, el rendimiento, la actividad física 
y la salud es la composición corporal (Robergs y Roberts, 2000). Esta ha sido ampliamente estudiada en la población general, dado que sus mediciones permiten cuantificar los mayores componentes estructurales del cuerpo humano, que a su vez tienen una fuerte relación con otras variables como por ejemplo, la condición física y el nivel de ejecución motriz (McArdle, Katch, y Katch,1996; Mayhew, 1981; Wilmore y Costill, 1999). El ejercicio produce cambios evidentes en la morfología humana, quedando también demostrada la modificación de la composición corporal mediante diferentes tipos de ejercicios (Benhke y Wilmore, 1974; Wilmore y Costill, 1999).

Existen modelos complejos para la determinación de la composición corporal, los cuales generalmente utilizan equipo sofisticado y costoso. Sin embargo, uno de los más utilizados se basa en la estimación de la masa grasa y de la masa libre de grasa (Robergs y Roberts, 2000; Yasumura, Wang, y Pierson, 2000).

La preparación física de un bailarín, especialmente de alto rendimiento, incluye ejercicios que le permiten desarrollar su masa muscular, con la consecuente reducción de la masa grasa. El exceso de grasa reduciría su capacidad de trabajo, ya que los requerimientos energéticos aumentarían al tener que trasladar mayor peso durante las ejecuciones.

La composición corporal de los bailarines no ha sido estudiada en Costa Rica, sin embargo, sí existen referencias de otros países. Por ejemplo, en USA, Dolgener, Spasoff y St. John (1980), midieron una muestra de bailarinas de ballet $(\mathrm{n}=19) \mathrm{y}$ de danza moderna $(n=10)$, entre 23 y 25 años y reportaron promedios en los porcentajes de grasa corporal (\% GC) de $22.1 \pm$ 0.35 en las bailarinas de ballet y de $22.1 \pm$ 0.66 en las bailarinas de danza moderna.

En un estudio de mujeres universitarias, Chambers (1981) seleccionó 5 bailarinas de nivel avanzado y 5 deportistas, con el fin de investigar los efectos de la danza en algunos parámetros de condición física como la composición corporal, la flexibilidad y la fuerza. El investigador reportó un \% GC = $12.7 \pm 1.74$ en las bailarinas, mientras que en las deportistas el \% $\mathrm{GC}=13.4 \pm 1.74$, aunque estas diferencias no fueron estadísticamente significativas.

Por otro lado, Clarkson, Freedson, Keller, Carney, y Skrinar (1985), administraron pruebas de composición corporal y capacidad aeróbica máxima a 14 bailarinas clásicas entre los 12-17 años de edad. Clarkson y colaboradores (1985) reportaron un \% GC = $16.4 \pm 4.0$ por medio del método de pesaje hidrostático.

Recientemente, Misigoj-Durakovic, Matkovic, Ruzic, Durakovic, Babic, Jankovic, y Ivancic-Kosuta (2001), estudiaron a 30 bailarinas profesionales del Croatian National Theatre Ballet. Los investigadores reportaron menores \% GC y mayor fuerza en el tren superior en las bailarinas solistas $(n=15)$ al compararlas con las demás miembros del elenco artístico $(n=15)$.

Aunque se han realizado estudios en bailarines profesionales y aficionados en otros países, no existen normas que permitan comparar diferentes categorías en variables de la composición corporal.

En Costa Rica, por ejemplo, existen normas de aptitud física y composición corporal para otras poblaciones (Fernández, González, Moncada, Pearson, Picado, y Salas, 2001). Sin embargo, hasta el momento no se han desarrollado normas específicamente para bailarines, las cuales son necesarias ya que se podrían establecer parámetros para determinar el estado de salud general del practicante y ofrecer a los directores de las diversas compañías de danza herramientas objetivas para una adecuada selección de candidatos. Así mismo, si las normas se emplean correctamente, se puede detectar casos de bailarines que requieren de intervenciones nutricionales o programas específicos de entrenamiento para mejorar, por ejemplo, su peso corporal. 
Por lo tanto, el objetivo de esta investigación fue establecer normas de composición corporal para el bailarín costarricense de danza contemporánea, tanto del sexo masculino como del femenino.

\section{Metodología}

\section{Participantes}

Se escogió la población de bailarines costarricenses de danza contemporánea. Estos sujetos debían ser reconocidos y aceptados como bailarines por los directores artísticos de las diferentes compañías de danza contemporánea del país; por lo tanto la categoría a la que pertenecían estaba establecida a priori. Así, se realizaron mediciones a 91 bailarines costarricenses de danza contemporánea, divididos en categoría profesional $(\mathrm{P})(\mathrm{n}=43)$, semiprofesional (SP) ( $\mathrm{n}=32)$, y aficionado (A) ( $\mathrm{n}=$ 16). Además, los participantes fueron agrupados según su género y rango de edad (17 a 21; 22 a 26; 27 a 31 y de 3235 años).

\section{Instrumentos de medición}

Para las mediciones antropométricas y de composición corporal se utilizaron los siguientes instrumentos. El peso se determinó con una balanza de resortes marca Health o Meter calibrada en kg. Para medir la talla corporal se empleó una cinta métrica de $0,5 \mathrm{~cm}$ de ancho, flexible e inextensible, calibrada en centímetros, con capacidad de dos metros de longitud e intervalos de 1,0 mm. Para la determinación de los pliegues subcutáneos se utilizó un calibrador de grasa marca Lange $\AA$, con una presión constante de $10 \mathrm{~g} \cdot \mathrm{mm}^{2}$.

\section{Procedimientos}

Antes de realizar el estudio con los bailarines, las investigadoras realizaron un plan piloto en el que midieron a 90 sujetos con el propósito de obtener valores confiables y válidos de las mediciones antropométricas que se aplicarían.

Luego del plan piloto, se citó a los participantes en su respectiva compañía de danza para realizarles las mediciones. A los bailarines se les especificó la hora y fecha de su medición, así como de la importancia del cumplimiento de los requisitos de medición, siguiendo las recomendaciones de Golding, Myers y Wayne (1982), y Jackson y Pollock (1985).

Para las mediciones de peso y talla se siguieron procedimientos estándares (Jackson y Pollock, 1985). Para la estimación de los pliegues se procedió a realizar las mediciones en el lado derecho del cuerpo. En las mujeres se midieron los pliegues del tríceps, subescapular, suprailíaco, abdominal, muslo y pantorrilla. En los hombres se midieron los pliegues anteriormente citados, más el pliegue del pecho, siguiendo las recomendaciones citadas por Jackson y Pollock (1985) para controlar la técnica.

Para obtener los valores cuantitativos de la composición corporal se utilizaron las ecuaciones de regresión generalizadas para cada sexo desarrolladas por Jackson y Pollock (1985), en las que primero se debe obtener el valor de la densidad corporal (DC) para posteriormente estimar el porcentaje de grasa corporal (\% GC) aplicando la fórmula de Siri (1956). Finalmente, se obtuvo el peso libre de grasa (PLG) y el peso de la grasa (PGR).

\section{Análisis estadístico}

Se analizaron las variables antropométricas y de composición corporal cuantitativas, a saber, peso $(\mathrm{kg})$, talla $(\mathrm{cm})$, pliegues subcutáneos (mm), \% GC, PLG y PGR. Para ello, se utilizó estadística descriptiva y análisis de varianza (ANOVA) de 1 vía para grupos independientes, únicamente en las mujeres. Los análisis de seguimiento se realizaron por medio del post hoc de Scheffé cuando se encontraron diferencias significativas 
en las pruebas de ANOVA. Finalmente, se utilizó una prueba $t$ student para muestras de diferente tamaño en los participantes del sexo masculino, para las categorías $\mathrm{P}$ y SP.

\section{Resultados}

Las características antropométricas y de composición corporal de los participantes del estudio se presentan en la tabla 1.

Como puede observarse en la tabla 1 , no se reportan los valores del pliegue del pecho en las mujeres, ya que el protocolo utilizado para obtener el \% GC no lo requería.

No se encontraron diferencias significativas en el peso o la talla en las mujeres de las distintas categorías. Sin embargo, por medio de la ANOVA de 1 vía para grupos independientes, se encontraron diferencias estadísticamente significativas $(\mathrm{p}<0.001)$ en el \% GC de las mujeres de las categorías $\mathrm{P}, \mathrm{SP}$, y A. El análisis post hoc de Scheffé indicó que las mujeres $\mathrm{P}$ mostraron menores \% GC que las mujeres de la categoría SP $(\mathrm{p}<0.05)$ y que las mujeres de la categoría A $(\mathrm{p}<0.001)$.

Por medio del ANOVA de 1 vía para grupos independientes, no se encontraron diferencias significativas en el peso libre de grasa, pero sí en el peso de la grasa $(\mathrm{p}<$ 0.005). El análisis post hoc de Scheffé indicó que el peso de la grasa de las mujeres de la categoría $\mathrm{P}$ era menor que el de las mujeres de la categoría A ( $\mathrm{p}<0.005)$.

Se comparó el peso, la talla, el \% GC, el peso libre de grasa y el peso de la grasa entre los hombres de las categorías $P$ y SP. La prueba $t$ de student para grupos independientes indicó que no existían diferencias estadísticamente significativas entre los promedios en ninguna de las variables estudiadas.

Se estudió cada variable en función de los rangos de edad solamente en las mujeres, ya que el número de hombres era insuficiente para agruparlos por edad. Se decidió agrupar a las mujeres en grupos

Tabla 1

Características físicas y de composición corporal de los participantes

\begin{tabular}{|c|c|c|c|c|c|c|}
\hline & \multicolumn{6}{|c|}{ Categoría } \\
\hline & \multicolumn{2}{|c|}{ Profesionales } & \multicolumn{2}{|c|}{ Semi-profesionales } & \multicolumn{2}{|c|}{ Aficionados } \\
\hline & $\begin{array}{c}\text { Femenino } \\
(\mathrm{n}=30)\end{array}$ & $\begin{array}{c}\text { Masculino } \\
(\mathrm{n}=13)\end{array}$ & $\begin{array}{l}\text { Femenino } \\
(\mathrm{n}=28)\end{array}$ & $\begin{array}{c}\text { Masculino } \\
(\mathrm{n}=4)\end{array}$ & $\begin{array}{c}\text { Femenino } \\
(\mathrm{n}=15)\end{array}$ & $\begin{array}{c}\text { Masculino } \\
\qquad(\mathrm{n}=1)\end{array}$ \\
\hline & $\mathrm{M} \pm \mathrm{DS}$ & $\mathrm{M} \pm \mathrm{DS}$ & $\mathrm{M} \pm \mathrm{DS}$ & $\mathrm{M} \pm \mathrm{DS}$ & $\mathrm{M} \pm \mathrm{DS}$ & Puntaje \\
\hline Edad (años) & $26 \pm 4$ & $26 \pm 3$ & $23 \pm 4$ & $24 \pm 3$ & $22 \pm 4$ & 25 \\
\hline Peso (kg) & $51,88 \pm 3,93$ & $64,58 \pm 5,04$ & $52,75 \pm 5,56$ & $61,83 \pm 5,08$ & $53,81 \pm 6,86$ & 66,00 \\
\hline Talla (cm) & $162,46 \pm 5,14$ & $173,19 \pm 3,54$ & $159,76 \pm 5,99$ & $170,40 \pm 5,46$ & $161,66 \pm 5,39$ & 173,00 \\
\hline Tríceps (mm) & $12,95 \pm 3,54$ & $8,35 \pm 2,97$ & $16,31 \pm 4,19$ & $7,13 \pm 0,75$ & $17,59 \pm 3,73$ & 10,20 \\
\hline Pecho (mm) & & $7,15 \pm 3,21$ & & $6,25 \pm 1,55$ & & 14,50 \\
\hline Sub-escapular (mm) & ) $10,01 \pm 2,19$ & $10,92 \pm 1,67$ & $2,24 \pm 3,47$ & $11,30 \pm 3,74$ & $12,11 \pm 3,07$ & 16,50 \\
\hline Suprailíaco (mm) & $7,52 \pm 3,40$ & $6,98 \pm 1,76$ & $10,12 \pm 5,26$ & $6,98 \pm 2,14$ & $11,58 \pm 4,63$ & 18,00 \\
\hline Abdominal (mm) & $9,78 \pm 4,66$ & $8,48 \pm 2,54$ & $3,07 \pm 6,61$ & $9,23 \pm 3,26$ & $13,27 \pm 3,77$ & 24,50 \\
\hline Muslo (mm) & $19,84 \pm 3,91$ & $10,19 \pm 3,73$ & $22,27 \pm 6,42$ & $7,80 \pm 0,96$ & $26,55 \pm 4,98$ & 19,50 \\
\hline$\%$ grasa & $17,16 \pm 3,14$ & $7,25 \pm 2,76$ & $19,77 \pm 4,54$ & $6,31 \pm 1,83$ & $22,14 \pm 3,46$ & 16,96 \\
\hline PLG (kg) & $42,95 \pm 3,20$ & $59,84 \pm 4,18$ & $42,20 \pm 3,89$ & $57,91 \pm 4,70$ & $41,77 \pm 4,51$ & 54,81 \\
\hline PGR (kg) & $8,93 \pm 1,90$ & $4,74 \pm 1,98$ & $10,55 \pm 3,16$ & $3,92 \pm 1,19$ & $12,04 \pm 3,14$ & 11,19 \\
\hline
\end{tabular}

Nota: $\mathrm{M}=$ Promedio; \pm Desviación estándar. 
etáreos de la siguiente manera: 17-21, 2226, 27-31 y 32-35. El ANOVA de una vía para grupos independientes indicó que no existían diferencias significativas en el peso, en la talla o en el peso libre de grasa; sin embargo, sí se encontraron diferencias estadísticamente significativas $(\mathrm{p}<0.05)$ en los promedios de \% GC y el peso de la grasa. El análisis post hoc de Scheffé indicó que las diferencias en los promedios de \% GC se encontraban en las mujeres de la categoría de 17-21 años con respecto a las categorías de 22-26 y 27-31 (Tabla 2).

Las normas porcentuales de las variables peso, talla, \% GC, PLG, y PGR para las diferentes categorías, fueron calculadas del 5 al $95 \%$ usando rangos de percentil (Tabla 3, 4, 5, 6 y 7).

Tabla 2

Estadística descriptiva de variables de composición corporal en bailarinas costarricenses

\begin{tabular}{lrrrr}
\hline & \multicolumn{5}{c}{ Grupos etáreos } \\
\cline { 2 - 5 } & $\begin{array}{c}17-21 \\
(\mathrm{n}=24)\end{array}$ & $\begin{array}{c}22-26 \\
(\mathrm{n}=30)\end{array}$ & $\begin{array}{c}27-31 \\
(\mathrm{n}=14)\end{array}$ & $\begin{array}{c}32-35 \\
(\mathrm{n}=5)\end{array}$ \\
\cline { 2 - 5 } & $\mathrm{M} \pm \mathrm{DS}$ & $\mathrm{M} \pm \mathrm{DS}$ & $\mathrm{M} \pm \mathrm{DS}$ & $\mathrm{M} \pm \mathrm{DS}$ \\
\hline & & & & $50,40 \pm 3,68$ \\
Peso $(\mathrm{kg})$ & $54,48 \pm 6,01$ & $52,29 \pm 4,28$ & $50,88 \pm 5,61$ & $162,58 \pm 3,33$ \\
Talla $(\mathrm{cm})$ & $160,90 \pm 5,45$ & $161,69 \pm 5,57$ & $160,51 \pm 6,79$ & $18,18 \pm 4,11$ \\
\% GC & $21,71 \pm 4,55$ & $18,01 \pm 3,64$ & $17,74 \pm 3,02$ & $41,21 \pm 3,22$ \\
PLG (kg) & $42,51 \pm 3,93$ & $42,84 \pm 3,51$ & $41,80 \pm 4,25$ & $9,19 \pm 2,36$ \\
\hline PGR (kg) & $11,97 \pm 3,43$ & $9,45 \pm 2,23$ & $9,08 \pm 2,19$ & \\
\hline
\end{tabular}

Nota: $\mathrm{M}=$ Promedio \pm \pm Desviación estándar.

Tabla 3

Normas de composición corporal para bailarinas profesionales costarricenses

\begin{tabular}{rccccc}
\hline Percentiles & Peso $(\mathrm{kg})$ & Talla $(\mathrm{cm})$ & \% GC & PLG $(\mathrm{kg})$ & PGR $(\mathrm{kg})$ \\
\hline 5 & 44,88 & 152,14 & 10,10 & 37,54 & 5,01 \\
10 & 47,23 & 155,14 & 12,71 & 38,86 & 5,86 \\
15 & 47,50 & 156,28 & 13,63 & 39,02 & 7,16 \\
20 & 47,80 & 157,02 & 15,29 & 39,57 & 7,58 \\
25 & 49,00 & 159,13 & 15,61 & 39,89 & 7,64 \\
30 & 49,50 & 160,36 & 16,08 & 40,81 & 7,81 \\
35 & 49,93 & 160,93 & 16,33 & 41,76 & 8,11 \\
40 & 50,50 & 161,98 & 16,86 & 42,15 & 8,71 \\
45 & 50,69 & 163,16 & 16,97 & 42,82 & 8,90 \\
50 & 51,75 & 163,65 & 17,22 & 43,08 & 8,95 \\
55 & 52,53 & 164,01 & 17,49 & 43,63 & 9,18 \\
60 & 53,34 & 164,38 & 18,05 & 43,97 & 9,72 \\
65 & 53,65 & 165,00 & 18,31 & 44,53 & 9,92 \\
70 & 54,50 & 165,00 & 18,90 & 44,56 & 9,95 \\
75 & 54,63 & 165,63 & 19,25 & 45,34 & 10,20 \\
80 & 55,80 & 166,72 & 19,49 & 46,02 & 10,62 \\
85 & 56,35 & 167,00 & 20,41 & 46,92 & 11,11 \\
90 & 57,00 & 169,25 & 21,52 & 47,60 & 11,63 \\
95 & 58,80 & 171,29 & 22,23 & 48,25 & 11,83 \\
\hline
\end{tabular}

Nota: \% GC: porcentaje de grasa corporal; PLG: peso libre de grasa; PGR: peso de la grasa. 
Tabla 4

Normas de composición corporal para bailarinas semi-profesionales costarricenses

\begin{tabular}{rccccc}
\hline Percentiles & Peso $(\mathrm{kg})$ & Talla $(\mathrm{cm})$ & $\%$ GC & PLG $(\mathrm{kg})$ & PGR $(\mathrm{kg})$ \\
\hline 5 & 43,72 & 148,78 & 13,76 & 35,72 & 6,26 \\
10 & 45,47 & 149,45 & 14,20 & 36,65 & 6,95 \\
15 & 46,39 & 152,61 & 14,36 & 38,90 & 7,31 \\
20 & 47,82 & 156,56 & 14,49 & 39,10 & 7,38 \\
25 & 48,68 & 157,13 & 15,13 & 39,26 & 7,63 \\
30 & 49,41 & 157,85 & 16,32 & 39,92 & 8,16 \\
35 & 50,58 & 158,02 & 17,57 & 40,26 & 9,13 \\
40 & 51,00 & 158,22 & 18,47 & 40,53 & 9,27 \\
45 & 51,53 & 158,62 & 18,98 & 40,95 & 9,43 \\
50 & 52,25 & 159,20 & 19,28 & 41,50 & 10,14 \\
55 & 52,98 & 159,88 & 20,11 & 42,19 & 10,60 \\
60 & 54,20 & 160,20 & 20,63 & 42,94 & 10,88 \\
65 & 54,50 & 161,48 & 21,72 & 43,56 & 11,00 \\
70 & 55,50 & 162,56 & 22,58 & 43,76 & 11,88 \\
75 & 55,88 & 162,78 & 23,05 & 45,12 & 13,37 \\
80 & 56,60 & 163,76 & 24,89 & 45,95 & 13,64 \\
85 & 59,28 & 168,23 & 25,07 & 46,93 & 14,57 \\
90 & 62,10 & 169,15 & 26,27 & 48,58 & 15,32 \\
95 & 64,10 & 170,61 & 28,43 & 49,86 & 17,01
\end{tabular}

Nota: \% GC: porcentaje de grasa corporal; PLG: peso libre de grasa; PGR: peso de la grasa.

Tabla 5

Normas de composición corporal para bailarinas aficionadas costarricenses

\begin{tabular}{cccccc}
\hline Percentiles & Peso $(\mathrm{kg})$ & Talla $(\mathrm{cm})$ & $\%$ GC & PLG $(\mathrm{kg})$ & PGR $(\mathrm{kg})$ \\
\hline 5 & 42,50 & 150,50 & 17,10 & 33,79 & 8,71 \\
10 & 44,72 & 152,12 & 17,53 & 34,64 & 8,72 \\
15 & 46,72 & 154,92 & 17,88 & 36,19 & 8,93 \\
20 & 47,70 & 157,70 & 18,49 & 37,91 & 9,26 \\
25 & 48,50 & 158,50 & 20,48 & 38,78 & 9,35 \\
30 & 49,30 & 159,22 & 20,62 & 39,58 & 9,72 \\
35 & 51,30 & 160,06 & 20,80 & 40,44 & 10,36 \\
40 & 52,70 & 160,70 & 20,98 & 40,99 & 10,84 \\
45 & 53,10 & 161,20 & 21,23 & 41,32 & 11,02 \\
50 & 53,50 & 162,00 & 21,66 & 41,93 & 11,08 \\
55 & 53,90 & 162,80 & 23,11 & 42,18 & 11,68 \\
60 & 54,00 & 163,06 & 23,54 & 42,79 & 12,30 \\
65 & 54,48 & 164,06 & 23,66 & 43,34 & 12,71 \\
70 & 55,36 & 165,52 & 23,77 & 43,73 & 12,85 \\
75 & 56,00 & 165,60 & 23,82 & 44,18 & 12,95 \\
80 & 61,76 & 166,08 & 23,87 & 44,65 & 14,91 \\
85 & 63,98 & 167,88 & 24,52 & 47,37 & 16,19 \\
90 & 65,50 & 169,36 & 27,36 & 49,57 & 17,86 \\
95 & 67,00 & 169,90 & 30,99 & 50,29 & 19,58
\end{tabular}

Nota: \% GC: porcentaje de grasa corporal; PLG: peso libre de grasa; PGR: peso de la grasa. 
Tabla 6

Normas de composición corporal para bailarines profesionales costarricenses

\begin{tabular}{rccccc}
\hline Percentiles & Peso $(\mathrm{kg})$ & Talla $(\mathrm{cm})$ & $\%$ GC & PLG $(\mathrm{kg})$ & PGR $(\mathrm{kg})$ \\
\hline 5 & 58,00 & 168,50 & 3,44 & 54,82 & 2,07 \\
10 & 58,60 & 169,06 & 3,88 & 55,19 & 2,34 \\
15 & 59,55 & 169,91 & 4,58 & 55,82 & 2,79 \\
20 & 59,90 & 169,98 & 4,95 & 56,35 & 2,96 \\
25 & 60,50 & 170,50 & 5,27 & 57,00 & 3,09 \\
30 & 61,10 & 171,30 & 5,51 & 57,59 & 3,25 \\
35 & 61,45 & 172,35 & 5,61 & 57,89 & 3,51 \\
40 & 62,40 & 172,68 & 5,76 & 57,95 & 3,81 \\
45 & 63,00 & 172,86 & 6,24 & 58,04 & 4,20 \\
50 & 63,00 & 173,00 & 7,14 & 58,24 & 4,72 \\
55 & 63,70 & 173,21 & 7,30 & 58,97 & 4,94 \\
60 & 64,80 & 173,58 & 7,51 & 59,35 & 5,04 \\
65 & 66,20 & 174,02 & 7,75 & 59,69 & 5,12 \\
70 & 67,60 & 174,16 & 7,95 & 61,31 & 5,62 \\
75 & 69,00 & 174,35 & 8,69 & 62,90 & 5,78 \\
80 & 70,10 & 174,56 & 9,84 & 64,31 & 6,28 \\
85 & 70,45 & 174,77 & 11,52 & 65,32 & 7,99 \\
90 & 73,20 & 179,72 & 12,43 & 67,71 & 8,39 \\
95 & 75,00 & 183,00 & 12,87 & 69,21 & 8,50 \\
\hline
\end{tabular}

Nota: \% GC: porcentaje de grasa corporal; PLG: peso libre de grasa; PGR: peso de la grasa.

Tabla 7

Normas de composición corporal para bailarines semi-profesionales costarricenses

\begin{tabular}{rccccc}
\hline Percentiles & Peso $(\mathrm{kg})$ & Talla $(\mathrm{cm})$ & $\%$ GC & PLG $(\mathrm{kg})$ & PGR $(\mathrm{kg})$ \\
\hline 5 & 57,00 & 163,00 & 4,47 & 54,45 & 2,55 \\
10 & 57,00 & 163,00 & 4,47 & 54,45 & 2,55 \\
15 & 57,00 & 163,00 & 4,47 & 54,45 & 2,55 \\
20 & 57,00 & 163,00 & 4,47 & 54,45 & 2,55 \\
25 & 57,88 & 164,85 & 4,82 & 54,70 & 2,80 \\
30 & 58,75 & 166,70 & 5,16 & 54,94 & 3,04 \\
35 & 59,63 & 168,55 & 5,50 & 55,18 & 3,29 \\
40 & 60,50 & 170,40 & 5,85 & 55,43 & 3,54 \\
45 & 60,58 & 170,85 & 5,91 & 55,81 & 3,70 \\
50 & 60,65 & 171,30 & 5,97 & 56,20 & 3,87 \\
55 & 60,73 & 171,75 & 6,04 & 56,58 & 4,04 \\
60 & 60,80 & 172,20 & 6,10 & 56,96 & 4,21 \\
65 & 62,85 & 173,15 & 6,78 & 58,92 & 4,50 \\
70 & 64,90 & 174,10 & 7,47 & 60,88 & 4,79 \\
75 & 66,95 & 175,05 & 8,15 & 62,83 & 5,08 \\
80 & 69,00 & 176,00 & 8,84 & 64,79 & 5,37 \\
85 & 69,00 & 176,00 & 8,84 & 64,79 & 5,37 \\
90 & 69,00 & 176,00 & 8,84 & 64,79 & 5,37 \\
95 & 69,00 & 176,00 & 8,84 & 64,79 & 5,37
\end{tabular}

Nota: \% GC: porcentaje de grasa corporal; PLG: peso libre de grasa; PGR: peso de la grasa. 


\section{Discusión}

Los resultados del presente estudio indican que el promedio de \% GC de las mujeres profesionales fue de $17,16 \%$, mientras que Chambers (1981) encontró un promedio de $12,7 \%$ GC en bailarinas profesionales. Por otro lado, Clarkson y otros (1985) encontraron un promedio de 16,9\% GC en bailarinas semiprofesionales, mientras que en el presente estudio fue de 19,77 $\%$ GC en bailarinas de la misma categoría. También se han obtenido promedios del $22,1 \%$ GC en bailarinas de danza clásica y moderna, aunque no se ha especificado la categoría (Dolgener y otros 1980).

$\mathrm{Al}$ comparar el \% GC entre las tres categorías; es decir, profesionales, semi-profesionales y aficionadas, se obtuvieron diferencias significativas entre las bailarinas profesionales con respecto a las semiprofesionales y a las aficionadas. Las mujeres profesionales presentaron \% GC menores que las mujeres de las otras dos categorías, lo cual se puede explicar por el número de horas de práctica diaria, ya que en promedio, las bailarinas profesionales practican 7 horas, las semi-profesionales 4 horas, y las aficionadas únicamente 1.5 horas. Con base en los resultados obtenidos en este estudio se observa claramente que aunque el peso promedio entre las bailarinas de las distintas categorías no cambia, sí lo hace la composición del mismo; es decir, a mayor grado de profesionalismo hay una tendencia a poseer menor cantidad relativa de grasa corporal. En otros estudios reportan diferencias entre las bailarinas de nivel avanzado y las de mediano nivel o sedentarias (Novak, Magill, y Schutte, 1978; Kuno, Fukunaga, Hirano, y Miyashita, 1996.

Un hallazgo que merece atención es que en este estudio también se encontró que las bailarinas de las diferentes categorías presentaron PLG similares; es decir, cantidades relativas similares de tejido muscular, óseo, agua, y otros. La relevancia de este hallazgo radica en que al menos en las edades de las bailarinas estudiadas, el grado de profesionalismo no es determinante de toda la composición corporal, sino únicamente de la cantidad relativa de grasa corporal. Se puede deducir entonces que existen otros factores, aparte del número de horas diarias de práctica, que hacen que se produzcan los cambios en el \% GC.

El contenido del tejido adiposo aumenta con la edad (Roche, 1984, 1987). Sin embargo, en esta investigación se encontró que en las mujeres, la cantidad proporcional de tejido adiposo disminuye significativamente. Las mujeres más jóvenes (17-21 años) presentaron \%GC mayores que los de las mujeres de 22-26 años y las de 27-31 años. De lo anterior, se deduce claramente que el \% GC de las bailarinas es menor que el de otras mujeres. Estas diferencias concuerdan con lo reportado previamente por otros (Novak et al., 1978; Kuno et al., 1996). Por ejemplo, Kuno et al. (1996), compararon los \% GC de bailarinas profesionales con los de mujeres sedentarias de la misma edad, encontrando \% GC y de peso corporal menores en las bailarinas que en sus contrapartes sedentarias.

De lo anterior se puede afirmar que tanto la categoría como el grado de actividad física sistemática pueden ser los responsables de los resultados obtenidos en el porcentaje de grasa y no necesariamente la edad, como algunos autores lo plantean, lo cual sugiere que no hay una relación directa entre el porcentaje de grasa y la edad, al menos en las bailarinas estudiadas. Además, existen diferencias raciales y/o étnicas que también hacen que los resultados obtenidos en la población anglosajona no sean comparables (Heyward y Stolarczyk, 1996).

Otro aspecto que merece discusión, es la metodología y la instrumentación utilizada para determinar la composición corporal. Actualmente, se cuenta con dispositivos más sensibles y con menor grado de error en la predicción que la estimación por el método de los pliegues cutáneos. Se 
debe recordar que el \% GC se estima, y no se mide directamente ya que es imposible en seres vivos. De esta forma, se desea que la estimación sea lo más acertada posible. Aunque se han desarrollado nuevas ecuaciones para la predicción de la composición corporal (Hergenroeder, Brown, y Klish, 1993; Yannakoulia, Keramopoulos, Tsakalakos, y Matalas, 2000), los instrumentos para obtenerla tienen un elevado costo, lo cual los hace prácticamente inaccesibles a muchas personas, por lo que el método de los pliegues cutáneos sigue siendo el método más utilizado.

Con los datos obtenidos en este estudio se desarrollaron normas de composición corporal para bailarinas y bailarines costarricenses, agrupados de acuerdo con la categoría. Estas normas permiten valorar la ubicación de los sujetos en cada uno de los componentes establecidos para este estudio. El diagnóstico de los futuros bailarines o aspirantes de las compañías de danza, se puede realizar de manera objetiva con estas normas. También pueden ser útiles para detectar bailarines que comprometan su salud, por ejemplo con \% GC muy bajos para su edad y sexo.

Un aporte significativo de esta investigación es la descripción de las variables antropométricas y de composición corporal en bailarines masculinos, ya que anteriormente no se reportaban sus características.

Es importante conocer la composición corporal (\% GC) en bailarines, ya que éstos tienden a presentar valores menores a los recomendados para mantener una buena salud (Heyward y Stolarczyk, 1996). Por ejemplo, en el presente estudio se encontró que las mujeres profesionales tenían 14-20 $\%$ GC; que de acuerdo con Heyward y Stolarczyk (1996) podrían catalogarse como menores al promedio (\% GC = 9-22). Estos valores no son tan bajos como los reportados por Pierce y Daleng (1998) en bailarinas de élite (\% GC = 10-16), los cuales fueron considerados como "excelentes". Generalmente, estos bajos \% GC se asocian con disturbios psicológicos (Pierce, y Daleng, 1998) y fisiológicos a mediano y largo plazo (To, Wong, y Chan, 1997).

Esta investigación es relevante para los bailarines, educadores e investigadores que se interesan por el estudio de la composición corporal, así como para estudiantes de disciplinas afines. Sirve, a su vez para comparar, periódicamente, la composición corporal que presenta cada bailarín con los estándares establecidos para otros bailarines a nivel mundial y para realizar comparaciones tanto inter como extra grupales, además como elemento de juicio objetivo para los dirigentes de las diversas compañías de danza al seleccionar futuros bailarines.

Las personas encargadas del reclutamiento y selección de los bailarines deben conocer el desarrollo humano en sus múltiples dimensiones. También debe convertirse en un educador que sea capaz de evaluar las necesidades físicas de los bailarines que está formando y ser capaz de planificar entrenamientos que cumplan con los objetivos propios de la danza, y que a la vez contribuyan con una salud adecuada. Para ello, es indispensable integrar a educadores y profesionales del movimiento humano y la salud a sus equipos de trabajo, ya que de las investigaciones revisadas para la elaboración de este manuscrito, se observó claramente que las bailarinas presentan indicadores de salud inadecuados, lo cual, desde todo punto de vista, debe corregirse.

\section{Referencias bibliográficas}

Benhke, A. R., y Wilmore, J. H. Evaluation and regulation of body build and composition. Englewood Cliffs, NJ: Prentice-Hall, 1974.

Bougart, M. Técnica de la danza (5ta ed.). Buenos Aires: Editorial Universal, 1966. 
Chambers, B. "Fitness of dancers and varsity athletes". Journal of Physical Education, Recreation, and Dance, 52(5), 46,49, 1981.

Clarkson, P., Freedson, P., Keller, B., Carney, D., y Skrinar, M. "Maximal oxygen uptake, nutritional patterns and body composition of adolescent female ballet dancers". Research Quarterly for Exercise and Sport, 56(2), 180-184, 1985.

Dolgener, F., Spasoff, T., y St. John, W. "Body build and body composition of high ability female dancers". Research Quarterly for Exercise and Sport, 51(4), 599-607, 1980.

Dumond, T. El ballet. Barcelona: Editorial Fama, 1954.

Fernández, A., González, C. Moncada, J., Pearson, G., Picado, M., y Salas, R. Normas Nacionales. Componentes de Salud Física: estudiantes costarricenses entre 8 y 17 años. San José, Costa Rica: Editorial Baula, 2001.

Golding, L., Myers, C., y Wayne, S. The Y's way to physical fitness (A guide for instructos). Chicago, IL: National Books of YMCA, 1982.

Hergenroeder, A. C., Brown, B., y Klish, W. J. "Anthropometric measurements and estimating body composition in ballet dancers". Medicine and Science in Sports and Exercise, 25(1), 145150, 1993.

Heyward, V. H., y Stolarczyk, L. M. Applied human body composition assessment. Champaign, IL: Human Kinetics, 1996.

Jackson, A., y Pollock, M. "Practical assessment of body composition". The
Physician and SportsMedicine, 13(5), 76-90, 1985.

Kuno, M., Fukunaga, T., Hirano, Y., y Miyashita, M. "Anthropometric variables and muscle properties of Japanese female ballet dancers". International Journal of Sports Medicine, 17(2), 100-105, 1996.

Mayhew, J. "Body composition”. American Journal of Physical Education, Recreation, and Dance, 52(7), 38-41, 1981.

McArdle, W., Katch, V., y Katch, F. Exercise physiology: Energy, nutrition, and human performance (3a. ed.). Philadelphia, PA: Lea \& Febiger, 1996.

Minton, S., y Beckwith, B. "Dance and sport". Journal of Physical Education, Recreation, and Dance, 57(5), 26,54, 1986.

Misigoj-Durakovic, M., Matkovic, B. R., Ruzic, L., Durakovic, Z., Babic, Z., Jankovic, S., y Ivancic-Kosuta, M. "Body composition and functional abilities in terms of the quality of professional ballerinas". Collegium Antropologicum, 25(2), 585-590, 2001.

Morehouse, L. Fisiología del ejercicio. Buenos Aires: Editorial El Ateneo, 1978.

Novak, L. P., Magill, L. A., y Schutte, J. E. "Maximal oxygen intake and body composition of female dancers". European Journal of Applied Physiology and Occupational Physiology, 39(4), 277-282, 1978.

Pierce, E. F., y Daleng, M. L. "Distortion of body image among elite female dancers". Perceptual and Motor Skills, 87(3 Pt 1), 769-770, 1998. 
Robergs, R. A., y Roberts, S. O. Fundamental principles of exercise physiology for fitness, performance, and health. Boston, MA: McGraw-Hill, 2000.

Roche, A. "The need for improvements in the measurement of body composition". American Journal of Physical Anthropology, 52(9), 256, 1984.

Roche, A. "Some aspects of the criterion methods for the measurement of body composition". Human Biology, 59(2), 209-220, 1987.

Siri, W.E. Gross composition of the body. En Lawrence, J. H., y Tobias, C. A. (Eds.), Advances in Biological and Medical Physics, vol. IV. New York, NY: Academic Press, Inc., 1956.

To, W. W., Wong, M. W., y Chan, K. M. “Association between body composition and menstrual dysfunction in collegiate dance students". The Journal of Obstetrics and Gynecology Research, 23(6), 529-535, 1997.

Wilmore, J. H., y Costill, D. L. Physiology of sport and exercise (2nd. Ed.). Champaign, IL: Human Kinetics, 1999.

Yannakoulia, M., Keramopoulos, A., Tsakalakos, N., y Matalas, A. L.. "Body composition in dancers: the bioelectrical impedance method". Medicine and Science in Sports and Exercise", 32(1), 228-234, 2000.

Yasumura, S., Wang, J., y Pierson, R. N. In vivo composition studies. Annals of the New York Academy of Sciences. Vol. 904. New York, NY: New York Academy of Sciences, 2000 .
Yamileth Chacón Araya Docente de la Escuela de Educación Física y Deportes Universidad de Costa Rica

Rocío Valverde Hidalgo Docente de la Escuela de Educación Física y Deportes Universidad de Costa Rica 
